\title{
Creating placebo responders and nonresponders in the laboratory: boons and banes
}

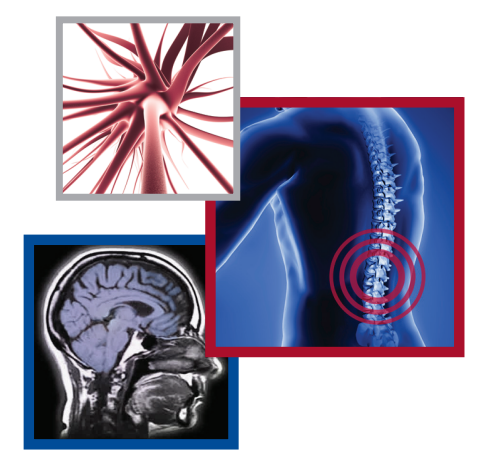

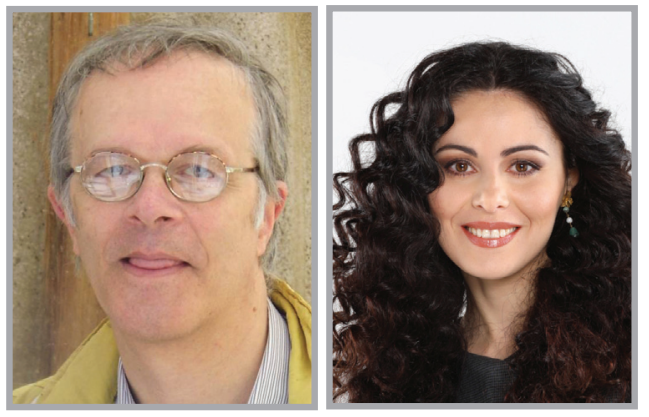

Fabrizio Benedetti, ${ }^{*}$ \& Elisa Frisaldi ${ }^{1}$

\author{
“Today we can manipulate these responses in \\ both directions, namely, we can create placebo \\ responders or nonresponders, particularly \\ in pain.”
}

On the basis of the recent insights into the mechanisms of the placebo response, particularly those involved in placebo analgesia, it is possible today to better identify some possible translational implications both within the context of medical practice and within the setting of clinical trials. Whereas in the former case we want to boost placebo responses, in the latter case we need to reduce them. Today we can manipulate these responses in both directions, namely, we can create placebo responders or nonresponders, particularly in pain. However, boons and banes need to be discussed from both a methodological and an ethical perspective. For example, it is important to remember that the improvement that may take place in a patient who has received a placebo may depend on plenty of factors, including spontaneous remission, regression to the mean, methodological biases, such as the experimenter's biased measurements and the patient's biased reports. In addition, the real psychological effect, which is attributable to both expectation and learning mechanisms, may also occur.

First and foremost, spontaneous remission, regression to the mean and methodological biases need to be done away with. Spontaneous remission can be eliminated by considering only those conditions and symptoms, like pain, whose natural history is well known. For example, it is very difficult to eliminate spontaneous pain fluctuations in chronic conditions such as headache, whereas the natural history of postoperative pain is pretty straightforward in most cases. Regression to the mean, which mainly depends on selection biases when recruiting patients in a clinical trial, can be controlled through strict and appropriate inclusion and exclusion criteria. Methodological biases can be reduced by using objective measurements in order to control both experimenter's and patient's biases.

Once all these factors are controlled and kept constant, some psychobiological placebo mechanisms can be used to enhance the placebo response. For example, learning plays a critical role in placebo responsiveness, so that subjects can be trained to respond. Indeed, not everyone responds to a placebo, especially if it is given for the first time; placebo analgesia is more robust when preconditioning with analgesic treatments is performed. This holds true for

\section{KEYWORDS}

- clinical trials • conditioning

- learning • medical practice

- methodological biases • natural history $\bullet$ placebo $\bullet$ regression to the mean 
both pharmacological and nonpharmacological treatments. In fact, a pharmacological preconditioning, whereby a painkiller is administered for several days in a row and then replaced with a placebo, has been found to increase the magnitude of placebo analgesia compared with a placebo given for the first time to naïve subjects [1]. Likewise, it is possible to use a nonpharmacological preconditioning paradigm in which the intensity of painful stimulation is reduced unbeknownst to the subjects, so as to make them believe that a treatment is effective. In this way, substantial placebo responses can be obtained after minutes, and these responses last up to 4-7 days. If the same conditioning procedure is performed in a group of subjects 4-7 days after an ineffective analgesic treatment, the placebo analgesic response is significantly reduced in magnitude [2]. Thus, both small and large placebo responses can be elicited, which indicates that the magnitude of the responses can be controlled by means of learning procedures. The underlying mechanism is likely to be represented by the reinforcement of positive expectations following the administration of repeated effective treatments. To support the important role of reinforced expectations, social observational learning has been found to produce placebo responses that are similar to those induced by directly experiencing the benefit through a conditioning procedure [3]. In other words, it is not necessary to undergo a first-person effective therapy in order to become a good placebo responder; the mere observation of the positive effects in another person is sufficient to trigger the same placebo analgesic responses.

The boons of this approach in medical practice are represented by the possibility to reduce drug intake in some circumstances. If drugs and placebos are administered alternately, the placebo mechanisms of conditioning and reinforced expectations can be harnessed to the patient's advantage, so that in the long run the intake of some drugs can be reduced along with some possible adverse events [4].

Although the possibility to manipulate the placebo response by using learning procedures can at first sight be greeted with enthusiasm by clinical trialists and drug companies, there are probably more banes than boons within these contexts, where the main interest is to reduce, or even abolish, the placebo response, so as to better evidence the specific effect of the active treatment. Indeed, it is possible to do this in a number of ways. The classical blockade of placebo analgesic responses has been obtained pharmacologically by means of opioid antagonists, such as naloxone [5], and cholecystokinin agonists, such as pentagastrin [6]. Today we know that naloxone blocks a descending pain modulatory network [7]. Although these studies show that placebo analgesia can be blocked by pharmacological agonists and antagonists of different neurochemical systems, their administration in a clinical trial with the purpose to reduce placebo responses is certainly unfeasible for a variety of reasons, first and foremost, their possible interference with the treatment under test.

Other somewhat different approaches seem to be more promising. For example, on the basis of the crucial involvement of the prefrontal lobes in the placebo response, repetitive transcranial magnetic stimulation (rTMS) has been used to inactivate the prefrontal cortex, and this inactivation has been found to disrupt placebo analgesia [8]. In addition, by using a negative conditioning procedure, whereby there is a mismatch between what the patient expects and what he gets, it is possible to reduce, and even completely abolish, placebo analgesia. It is also worth mentioning that some genotypes related to high or poor responsiveness to placebos have been identified in medical conditions such as major depression [9], social anxiety [10], irritable bowel syndrome [11] and pain [12], thus hinting at a possible future genetic screening before enrolment in a clinical trial.

Despite some enthusiasm regarding the possibility to reduce placebo responses in the near future, the main question is what use we make of this. From a methodological point of view, whether or not laboratory-created placebo nonresponders can be enrolled in a clinical trial remains an open question for at least four reasons. First, the global response to the active treatment would also be reduced. In fact, if we randomize placebo nonresponders to placebo and active treatment, we will witness a reduction of the response in both groups, with no real advantage compared with a classical trial. Second, the trial would not be representative of the general population, which means that the average response to the active treatment will be different when the medication will be on the market. Third, as already emphasized above, creating placebo nonresponders through negative conditioning or rTMS does not mean to also control natural history, regression to the 
mean, and methodological biases. Therefore, the recruitment of placebo nonresponders in trials in which the spontaneous fluctuation of symptoms is the rule, as occurs in chronic pain, would not be resolutive for this kind of approach. Fourth, although all this holds true for pain, it is not clear whether other medical conditions and symptoms are amenable to the same manipulation. At the present time, we know that Parkinson's disease represents another medical condition whose placebo mechanisms can be identified and analyzed [13], but we know much less about other ailments.

From an ethical point of view, a placebo procedure usually requires some kind of deception. Therefore, the manipulation of placebo responsiveness in the clinical trial setting can be viewed as highly questionable if the only objective is to reduce the placebo effect. The main point that needs to be discussed is whether or not these procedures are justified in patients who are enrolled in a clinical trial. For example, in some severe conditions, it would certainly be unethical to create placebo nonresponders only to demonstrate a bigger difference between active treatment and placebo groups.

In summary, we believe that it is important to emphasize how the improvement that may take place following the administration of a placebo depends on many factors. What we can do today is to manipulate only a part of these factors, such as the psychological component. However, while this manipulation may be effective within the context of medical practice, in a clinical trial many other factors may be responsible for the improvement, including the natural course of the disease or symptom, the appropriateness of the inclusion and exclusion criteria, the outcome measures and endpoints, and all these factors are not easily manageable. Therefore, although we are today in a good position to modulate the placebo response in both directions, and to create placebo responders or nonresponders in the laboratory by using a number of approaches, such as conditioning and learning procedures, the translational and ethical implications are far from being definitive and require an in-depth methodological and ethical discussion.

\section{Financial \& competing interests disclosure}

This work was supported by Compagnia di San Paolo Foundation and Carlo Molo Foundation. The authors have no other relevant affiliations or financial involvement with any organization or entity with a financial interest in or financial conflict with the subject matter or materials discussed in the manuscript apart from those disclosed.

No writing assistance was utilized in the production of this manuscript.

\section{References}

1 Benedetti F, Amanzio M, Rosato R, Blanchard C. Non-opioid placebo analgesia is mediated by CB1 cannabinoid receptors. Nat. Med. 17(10), 1228-1230 (2011).

2 Colloca L, Benedetti F. How prior experience shapes placebo analgesia. Pain 124(1), 126-133 (2006).

3 Colloca L, Benedetti F. Placebo analgesia induced by social observational learning. Pain 144(1), 28-34 (2009)

4 Doering BK, Rief W. Utilizing placebo mechanisms for dose reduction in pharmacotherapy. Trends Pharmacol. Sci. 33(3), 165-172 (2012).

5 Amanzio M, Benedetti F. Neuropharmacological dissection of placebo analgesia: expectation-activated opioid systems versus conditioning-activated specific subsystems. J. Neurosci. 19(1), 484-494 (1999).
6 Benedetti F, Amanzio M, Thoen W. Disruption of opioid-induced placebo responses by activation of cholecystokinin type-2 receptors. Psychopharmacology 213(4), 791-797 (2011)

7 Eippert F, Bingel U, Schoell ED et al. Activation of the opioidergic descending pain control system underlies placebo analgesia. Neuron 63(4), 533-543 (2009).

8 Krummenacher P, Candia V, Folkers G, Schedlowski M, Schönbächler G. Prefrontal cortex modulates placebo analgesia. Pain 148(3), 368-374 (2010).

9 Leuchter AF, McCracken JT, Hunter AM, Cook IA, Alpert JE. Monoamine oxidase a and catechol-O-methyltransferase functional polymorphisms and the placebo response in major depressive disorder. J. Clin. Psychopharmacol. 29(4), 372-377 (2009).

10 Furmark T, Appel L, Henningsson S et al. A link between serotonin-related gene polymorphisms, amygdala activity, and placebo-induced relief from social anxiety. J. Neurosci. 28(49), 13066-13074 (2008).

11 Hall KT, Kaptchuk TJ. Genetic biomarkers of placebo response: what could it mean for future trial design? Clin. Investig. 3(4), 311-314 (2013).

12 Peciña M, Martínez-Jauand M, Hodgkinson C, Stohler CS, Goldman D, Zubieta JK. FAAH selectively influences placebo effects. Mol. Psychiatry 19(3), 385-391 (2014).

13 Frisaldi E, Carlino E, Lopiano L, Lanotte M, Benedetti F. Characterization of the thalamic-subthalamic circuit involved in the placebo response through single-neuron recording in Parkinson patients. Cortex doi:10.1016/j.cortex.12.003 (2013) (Epub ahead of print). 